\title{
Ethical nuances and medicolegal vulnerabilities in robotic neurosurgery
}

\author{
George Chandy Vilanilam, FRCS, MCh, MS, DNB, MBA, MBBS, and \\ Easwer Hariharan Venkat, MCh, MBBS \\ Department of Neurosurgery, Sree Chitra Tirunal Institute for Medical Sciences and Technology, Trivandrum, Kerala, India
}

\begin{abstract}
Inter arma enim silent lēgēs (Latin) (In times of war, the laws
\end{abstract} fall silent).

$$
\text { - Cicero }
$$

Ethical values and medicolegal fidelity are deeply ingrained in our surgical culture. Yet, these are often put to an acid test by cutting-edge technologies like robotic surgery (RS), where ethical and legal proprieties may at times be in peril. ${ }^{1}$ Surgical endeavors are not just mechanical tasks, but a complex culmination of experience, anatomical familiarity, creativity, adaptability to variation, and emergency mayday measures, and are, thus, considered difficult to automate. ${ }^{1,2}$ As surgical sciences catch up with the robotic revolution, the caring, empathetic, compassionate surgeon faces the threat of being outclassed by a faster, stronger, more learned, and efficient machine. ${ }^{3-5}$ The spectrum of surgical possibilities in robotic competencies and levels of autonomy are rapidly expanding, creating moral, ethical, and medicolegal dilemmas and vulnerabilities. ${ }^{1,2}$

\section{Journey of a New Surgical Technology}

The arduous journey of a new surgical technology begins with early adopters who test it and excitedly market its benefits to the skeptics. The new technology may evolve further to become "one of the options," comparable with traditional surgical methods. ${ }^{4,5}$ Finally, when its safety, efficacy, and outcome standards have been established over and above other traditional methods, it becomes accepted as the standard of care.

Michael Brady defined robotics conceptually, as "the field concerned with the intelligent connection of perception to action." "Robota" (Czech for "servitude") refers to an "automated and programmed" help for humans. The term was coined by Karel Čapek (1920), a Czech playwright, and was popularized by Isaac Asimov's science fiction. ${ }^{7,8}$ The Neuromate (Integrated Surgical Systems, 1999), used to perform stereotactic brain biopsy, was one of the earliest surgical robots. The spectrum of robotic neurosurgery has expanded to include skull base endos- copy, depth electrode placement for chronic invasive EEG monitoring, deep brain stimulation, and skull base and spinal tumor resections with several levels of robotic autonomy. ${ }^{9,10}$ Superior dexterity, precision, and indefatigability, better stereoscopic vision, miniaturized access routes, and more degrees of freedom for surgical instruments are some of the benefits offered by the robotic arm. The complex intricacies of neurosurgery, with its narrow safety margin, make it a more difficult prospect for rapid adoption of RS. ${ }^{9-13}$ Perception (haptics), manipulation skills, creativity, and social intelligence are still considered human surgical territory. However, robotization of menial mechanical surgical tasks may help prioritize time for more "humanistic" skill-based tasks. ${ }^{2,3}$

\section{New Technology Bias}

From driverless cars to smartphone applications, new technology has an aura of sophistication. We subconsciously believe that the advantages of new technology outshine the accepted old methods, despite scarce evidence to support this view. ${ }^{14}$ The complexity of new surgical technology creates a bias conferring more credibility and legitimacy on it, more so, because technical algorithms are often considered to be beyond the realm of surgical sciences. ${ }^{8,14}$

As the extent of robotic autonomy is evolving quickly, several ethics concerns and medicolegal standards need reexamination. The concept of medical negligence rests on an established standard of care, falling short of it, and the resultant harm. The traditional paternalistic model of surgical care has given way to a more consumerist and technical model. ${ }^{15,16}$

\section{Ethical Nuances From a Robotic Neurosurgery Perspective}

Ethics encompasses a set of moral principles for the general good of the individual and society. The Nuremberg code (1947) and the Belmont report (1978) offer 
practical checklists to ensure the maintenance of ethical standards, as enumerated below.

\section{Autonomy}

Autonomy is the right of an individual to self-determination, which respects their ability to make informed decisions. When tempted by robotic technology and its potential benefits, an unbiased autonomous decision is challenging. The wide variety of educational and cultural backgrounds of patients (ethical relativism), constrained by limited and imperfect scientific information, make autonomous decision-making very tough. ${ }^{2,15}$ Media, internet, and industry proponents could further impair the autonomy of surgical decisions. Thus, robotic surgeons must be balanced, "matter of factly," and objective in the presentation of information without stifling autonomy. ${ }^{5}$

\section{Beneficence}

Beneficence is defined as an act of mercy, charity, and kindness, with a strong intent of doing good to others as a moral obligation. ${ }^{3}$ It is, thus, imperative to prescribe treatment where the benefits far outweigh procedural risks, with outcomes meeting acceptable standards. RS requires a new skill set and rigorous training; hence, adverse events and technical failures in the early part of the learning curve may exceed those of conventional surgery. ${ }^{4,7}$ It is crucial that surgeons, institutions, and professional bodies establish accreditation and training standards to ensure that RS measures up and evolves to accepted norms.

\section{Non Maleficence}

Non maleficence is the obligation of a surgeon to not cause harm, inadvertent or volitional. ${ }^{2,4}$ Despite the "good faith" involved, the long-term benefits and drawbacks of RS have not been clearly established or experienced. Harm and error could be mitigated by skill upgradation initiatives and reappraisals. Failed RS is often attributable to a system malfunction or inadequate experience. Transparent and open-access reporting of RS outcomes, as in the Manufacturer and User Facility Device Experience (MAUDE) database, could be invaluable to ensure nonmaleficence in care. ${ }^{16,17}$

\section{Justice}

Justice refers to everyone having an equal opportunity at therapeutic benefit. Thus, the benefits of new technology like RS need to be accorded without any bias or discrimination. ${ }^{3}$ A reverse bias that results in offering less-accepted or investigational surgical technology to economically challenged or resource-constrained patient populations in the guise of superior technology, also impairs the principle of justice. Dignity, truthfulness, and honesty in surgical care are the newer dimensions in surgical bioethics that one needs to be mindful of. ${ }^{2,3}$

\section{Medicolegal Vulnerabilities: The Robotic Neurosurgery Perspective}

A strong sense of justice and patient rights prevail in the practice of contemporary medicine. Evolving surgical therapies are less stringently governed in comparison to new medical devices, creating several gray areas in legal concepts. ${ }^{16,17}$

\section{Standard of Care}

The degree of care (attention, watchfulness, caution, and prudence) that a reasonable person should exercise while delivering medical care is defined as the standard of care. Current evidence and guidelines ensure its upkeep, thereby mitigating iatrogenic errors. ${ }^{2,15}$ Standards of care could vary across nations depending on socioeconomic factors, expertise, and infrastructure. As collective experience in RS is still scarce and evolving, it would not often meet the standard-of-care definition, thus inciting medicolegal liabilities.

\section{Medical Negligence and Tort Laws}

An act of omission that does harm to another and amounts to a civil wrong for which courts impose a liability is termed a tort. According to Charlesworth et al., the four constituents to establish medical negligence are existence of a duty of care, breach of duty, consequent damage, and a direct causal relationship., ${ }^{2,5,18}$ An error of commission or omission leading to a surgical adverse event during RS, beyond the acceptable norms, could be deemed as negligence. Failure to meet accepted surgical competency standards, inadequate robotic surgical experience, and machinery malfunction could all fall under the legal ambit of negligence..$^{2,15,19}$

\section{Surgical Responsibility}

Since several degrees of surgical autonomy of robots can be expected in the future, the extent of responsibility of the surgeon could vary. Yang et al. described five levels of robotic autonomy; these are level 0 (no autonomy), level 1 (robotic assistance), level 2 (task autonomy), level 3 (conditional autonomy), level 4 (high autonomy), and level 5 (full autonomy). ${ }^{2}$ "Meaningful human control" of increasingly autonomous surgical robots is a fastevolving concept, which ensures human responsibility and accountability while permitting limited forms of robotic autonomy. A surgeon's responsibility includes accountability, liability, and culpability. Accountability refers to the capacity of a system to provide an explanation for its actions. In fully autonomous robotic surgical systems, accountability would be shared between the surgical team and the device manufacturer. The credentialing professional bodies also share an element of accountability. Liability refers to accepting blame for inaction or wrong actions. Culpability includes punishment for wrongdoing; although, a robot cannot be punished as it has no concept of civil liberties. In the future, however, machine learning robots may include a mechanism to accumulate rewards and penalties..$^{2-4}$

\section{Informed Consent and Reasoned Refusal}

The concept of informed surgical consent denotes shared decision-making and abhors traditional paternalistic practices. ${ }^{5}$ Key issues in RS consent are as follows. 1) Has robotic surgical procedural safety been established beyond doubt? 2) Are the surgeons trained enough for proficient and safe usage? 3) How are the procedural 
outcomes evaluated and compared with time-tested standards?

The role of the proctor (the teacher responsible for the operation), the preceptor (the expert who validates a surgeon's competence), and the assistant, with each of their responsibilities clearly outlined, must be adequately informed. A "reasoned refusal" should not preclude a future consent, as informed consent is a process, rather than a one-time event. ${ }^{5}$

\section{Confidentiality and Cybersecurity}

Clinical data security concerns arise when third parties outside the treating team are involved. Surgeon and hospital data, especially those related to RS experiences, also need protection. Data and device hacking is a growing concern, as hackers could corrupt the robot's algorithms and machine learning patterns, with lethal surgical consequences. ${ }^{7,8}$

\section{Conflicts of Interest}

Expensive investments in RS create an intense need to protect its existence and promote its use. ${ }^{7}$ Traditional open surgical procedures may be projected unfairly as archaic and outdated in the growing enthusiasm to promote RS. Advertising and remuneration incentives may unfairly influence the unscientific trend of promoting RS well before its time..$^{8,19}$

\section{Litigation and Medical Malpractice Suits}

The MAUDE database (2000-2013) reported 144 deaths (1.4\% of the 10,624 reports), 1391 patient injuries (13.1\%), and 8061 device malfunctions (75.9\%). ${ }^{20}$ Litigation following cybersurgery will involve multiple defendants who could use finger-pointing defenses or unfair blame games. Hence, clear standards for fixing the responsibilities in RS should be defined. A "black box" of RS, as in an aircraft's safety system, would help in localizing the point of error and eventually enhance surgical quality. ${ }^{16}$

\section{Surgeon's Defense in RS Malpractice}

Charges of medical negligence, tort violations, or breach of contract could be part of RS lawsuits. The surgeon's defense is dependent on the level of the robotic autonomy. ${ }^{16}$ The surgeon, hospital, and device manufacturer should be part of the defense to avoid shift of blame and the "empty chair" defense. The manufacturer is also liable in machine malfunctions. Adverse events conforming to acceptable surgical quality standards and additional exigent circumstances during surgery could further absolve the surgeon. ${ }^{17}$ Updated robotic surgical accreditation and training standards strengthen the defense claim. In the event that RS was an option alongside traditional nonrobotic approaches, the strength of the defense lies in proving that acceptable robotic surgical standards were met and that all other options were discussed and offered without bias. ${ }^{17,19}$

\section{Future Directions}

\section{Evaluating New Technologies}

A robust tracking system for reporting adverse events in RS is an essential need in neurosurgery. Prospective and open reporting systems will allow surgeons and manufacturers to adapt more quickly. Post-market surveillance of robotic machines must be done by all the stakeholders including surgeons, hospitals, manufacturers, and the regulatory bodies. ${ }^{7,20}$ In certain specialties, a fundamentals of RS curriculum has been created and is currently undergoing a validation process. The evaluation and implementation of new techniques should be handled at a local, institutional level conforming to ethical, moral, and medicolegal standards. National organizations may provide guidance regarding credentialing standards, but granting of privileges to perform a procedure or use a technology remains the prerogative of the local institution. ${ }^{20}$

\section{Timing of Introduction and Striking a Balance}

Utmost care should be exercised in timing the introduction of new RS procedures, balancing the wait for sufficient supportive data with the needs of patients. ${ }^{8-10}$ Undue ethical concerns, delays, and bottlenecks may deprive patients of state-of-the-art care. New technology committees (NTCs), as the local regulatory authority in every institution, are desirable. NTCs are different from Institutional Review Boards, which maintain an overview of clinical research. NTCs are also expected to assure stabilization of techniques, coordinate efforts with patient safety personnel, manage potential conflicts of interest, and evaluate the impact on institutional resources. Market forces, societal pressures, and administrative exigencies must not compromise the ethical principles in the early adoption of RS..$^{7,20}$

\section{Roboethics}

Roboethics concerns the ethical use of robotic technology. This ensures that machines with artificial intelligence prioritize human safety over their assigned tasks and their own subsistence, in accordance with accepted precepts of human morality. Consequences of patient death or disability from a robotic surgical error would be within the roboethics ambit, akin to a fatal accident in a driverless car. $^{15,16,20}$

\section{Conclusions}

The adoption of state-of-art, yet nascent, robotic surgical techniques needs a tactful balance to uphold ethical, moral, and medicolegal standards of neurosurgical care. Misplaced ethical concerns and legal intricacies could stifle progress and stunt the growth of the robotic surgical spectrum. A reexamination of ethical concepts and medicolegal proprieties from an RS perspective could elevate standards of care and enhance outcomes, without an iota of ethical or medicolegal compromise.

https://thejns.org/doi/abs/10.3171/2021.10.FOCUS21533

\section{References}

1. Geiger JD, Hirschl RB. Innovation in surgical technology and techniques: challenges and ethical issues. Semin Pediatr Surg. 2015;24(3):115-121.

2. Yang GZ, Cambias J, Cleary K, Daimler E, Drake J, Dupont $\mathrm{PE}$, et al. Medical robotics-regulatory, ethical, and legal considerations for increasing levels of autonomy. Sci Robot. 2017;2(4):eaam8638. 
3. Vvedenskaya E. Bioethical aspects of robotics in surgery. JAHR. 2021;12(1):129-139.

4. Schwartz JA. Innovation in pediatric surgery: the surgical innovation continuum and the ETHICAL model. J Pediatr Surg. 2014;49(4):639-645.

5. Ferrarese A, Pozzi G, Borghi F, Pellegrino L, Di Lorenzo P, Amato B, et al. Informed consent in robotic surgery: quality of information and patient perception. Open Med (Wars). 2016;11(1):279-285.

6. Brady M. Artificial intelligence and robotics. Artif Intell. 1985;26(1):79-121.

7. O'Sullivan S, Nevejans N, Allen C, Blyth A, Leonard S, Pagallo U, et al. Legal, regulatory, and ethical frameworks for development of standards in artificial intelligence (AI) and autonomous robotic surgery. Int J Med Robot. 2019;15(1): e1968.

8. Strong VE, Forde KA, MacFadyen BV, Mellinger JD, Crookes PF, Sillin LF, Shadduck PP. Ethical considerations regarding the implementation of new technologies and techniques in surgery. Surg Endosc. 2014;28(8):2272-2276.

9. Wang MY, Goto T, Tessitore E, Veeravagu A. Introduction. Robotics in neurosurgery. Neurosurg Focus. 2017;42(5):E1.

10. Madhavan K, Kolcun JPG, Chieng LO, Wang MY. Augmented-reality integrated robotics in neurosurgery: are we there yet? Neurosurg Focus. 2017;42(5):E3.

11. Sutherland G. Introduction to virtual reality and robotics in neurosurgery. Neurosurgery. 2013;72(suppl 1):7.

12. Doulgeris JJ, Gonzalez-Blohm SA, Filis AK, Shea TM, Aghayev K, Vrionis FD. Robotics in neurosurgery: evolution, current challenges, and compromises. Cancer Contr. 2015; 22(3):352-359.

13. Mattei TA, Rodriguez AH, Sambhara D, Mendel E. Current state-of-the-art and future perspectives of robotic technology in neurosurgery. Neurosurg Rev. 2014;37(3):357-366.
14. MIT Sloan Management Review. Evaluating New Technology? You're More Biased Than You May Realize. Published September 23, 2020. Accessed November 4, 2021. https: //sloanreview.mit.edu/article/evaluating-new-technologyyoure-more-biased-than-you-may-realize/

15. Dickens BM, Cook RJ. Legal and ethical issues in telemedicine and robotics. Int J Gynaecol Obstet. 2006;94(1):73-78.

16. McLean T. The complexity of litigation associated with robotic surgery and cybersurgery. Int J Med Robot. 2007;3(1): 23-29.

17. Ferrarese A, Pozzi G, Borghi F, Marano A, Delbon P, Amato $\mathrm{B}$, et al. Malfunctions of robotic system in surgery: role and responsibility of surgeon in legal point of view. Open Med (Wars). 2016;11(1):286-291.

18. Charlesworth J, Todd S, Cooper R, Wood S, Walton C. Charlesworth \& Percy On Negligence. Sweet \& Maxwell; 2010.

19. McLean TR. The legal and economic forces that will shape the international market for cybersurgery. Int J Med Robot. 2006;2(4):293-298.

20. Alemzadeh H, Raman J, Leveson N, Kalbarczyk Z, Iyer RK. Adverse events in robotic surgery: a retrospective study of 14 years of FDA data. PLoS One. 2016;11(4):e0151470.

\section{Disclosures}

The authors report no conflict of interest.

\section{Correspondence}

George Chandy Vilanilam: gvi199@yahoo.com.

INCLUDE WHEN CITING

DOI: $10.3171 / 2021.10 . F O C U S 21533$. 